УДК 331.215-027.541:331.556.2

DOI dx.doi.org/10.24866/1813-3274/2020-1/46-60
А. Р. Нагапетян ${ }^{1}$, Дальневосточный федеральный университет,
г. Владивосток, Россия
E-mail: nagapetyan_ar@dvfu.ru
Д. В. Гришин ${ }^{2}$, Дальневосточный федеральный университет, г. Владивосток, Россия
E-mail: grishin.dv@students.dvfu.ru
А. В. Кедровская ${ }^{3}$, Дальневосточный федеральный университет, г. Владивосток, Россия
E-mail: kedrovskaia.av@students.dvfu.ru

\title{
ПРОСТРАНСТВЕННО-АВТОРЕГРЕССИОННЫЙ АНАЛИЗ МЕЖРЕГИОНАЛЬНОЙ ВЗАИМОСВЯЗАННОСТИ ЗАРАБОТНЫХ ПЛАТ В ОТРАСЛЕВОМ РАЗРЕЗЕ
}

Аннотация. Важной причиной оттока трудоспособного населения, помимо низкого уровня развитости инфраструктуры, является сравнительно более низкий уровень заработных плат по сравнению с другими регионами. Существует ли межрегиональная взаимосвязанность между величинами заработных плат? С одной стороны, возможно наличие положительной пространственной корреляции. Так, повышение оплаты труда в соседнем регионе приведет к тому, что у рабочих появятся стимулы к переезду в этот регион, что, в свою очередь, может привести к дефициту рабочих в рассматриваемом регионе, что, в свою очередь, создаст стимулы для местных компаний повысить оплату труда. С другой стороны, положительная пространственная корреляция может и не наблюдаться или быть незначимой, например, в случае наличия тех или иных барьеров, препятствующих переезду из

\footnotetext{
${ }^{1}$ Артур Рубикович Нагапетян ${ }^{1}$, старший преподаватель департамента экономических наук, Школа экономики и менеджмента, Дальневосточный федеральный университет, г. Владивосток, Россия.

2 Данил Витальевич Гришин, студент, Школа экономики и менеджмента, Дальневосточный федеральный университет, г. Владивосток, Россия.

${ }^{3}$ Анна Викторовна Кедровская, студент, Школа экономики и менеджмента, Дальневосточный федеральный университет, г. Владивосток, Россия.

Для цитирования: Нагапетян А. Р., Гришин Д. В., Кедровская А. В. Пространственно-авторегрессионный анализ межрегиональной взаимосвязанности заработных плат в отраслевом разрезе // Азиатско-Тихоокеанский регион: экономика, политика, право. 2020. № 1. С. 46-60.
}

(C) Нагапетян А. Р., Гришин Д. В., Кедровская А. В., 2020 
Нагапетян А. Р., Гришин Д. В., Кедровская А. В. Пространственно-авторегрессионный анализ межрегиональной взаимосвязанности заработных плат в отраслевом разрезе

одного региона в другой. В статье был произведен пространственноавторегрессионный анализ взаимосвязанности региональных заработных плат в отраслевом разрезе, позволяющий ответить на вопрос, увеличится или уменьшится величина заработной платы в регионе в конкретной отрасли при увеличении соответствующего показателя в соседних регионах при прочих равных условиях. В рамках моделирования было в том числе оценено влияние на заработную плату некоторых социально-экономических факторов. К их числу относятся уровень образованности, доля пенсионеров, коэффициент демографической нагрузки, уровень развития финансовой инфраструктуры в регионе, уровень безработицы, производительность труда. Основной новизной работы представляется использование моделей пространственной авторегрессии, что помимо выявления пространственных взаимосвязей позволило получить более точные оценки коэффициентов, характеризующих влияние на заработную плату рассмотренных факторов ввиду того, что частично удаётся решить известные проблемы, связанные с наличием пропущенных переменных в классических регрессионных моделях. Для анализа были рассмотрены соответствующие региональные данные с 2012 г. по 2016 г. для 83 субъектов Российских Федерации по следующим отраслям: сельское хозяйство, охота и лесное хозяйство; производство пищевых продуктов (включая напитки) и табака; строительство; оптовая торговля, включая торговлю через агентов; розничная торговля, государственное управление и военная безопасность, обработка древесины и производство изделий из дерева (кроме мебели). Положительная значимая пространственной корреляции была выявлена для всех рассмотренных отраслей, кроме оптовой торговли и обработки древесины. Полученные результаты и сформулированные выводы в дальнейшем могут применяться органами государственной власти при реализации мер социально-экономической политики, направленных на регулирование рынка труда, в том числе мер по привлечению и удержанию кадров на конкретной территории.

Ключевые слова: движение трудовых ресурсов, дифференциация доходов населения, модель с фиксированными эффектами, межрегиональная зависимость зарплат, модели с пространственными эффектами, реальная заработная плата, пространственная взаимосвязь, пространственная выборка, пространственноавторегрессионный анализ, пространственные эффекты, региональные отраслевые заработные платы, региональные факторы, социальная политика, экономическая безопасность страны. 
Artur R. Nagapetyan ${ }^{1}$, Far Eastern Federal University, Vladivostok, Russia

E-mail: nagapetyan_ar@dvfu.ru

Danil V. Grishin ${ }^{2}$, student, Far Eastern Federal University,

Vladivostok, Russia

E-mail: grishin.dv@students.dvfu.ru

Anna V. Kedrovskaya ${ }^{3}$, student, Far Eastern Federal University, Vladivostok, Russia

E-mail: kedrovskaia.av@students.dvfu.ru

\section{SPATIAL-AUTOREGRESSIVE ANALYSIS OF INTER-REGIONAL INTER- CONNECTEDNESS OF WAGES IN THE CONTEXT OF INDUSTRY}

Abstract. Besides the low level of infrastructural development, the lower level of wages in comparison with other regions is a major cause of labor force outflow. Is there any interregional interconnection between the levels of wages? On the one hand, there is a possibility for a positive spatial correlation. Thus, increase of wages in the neighboring region will result in the stimuli for workers to move into this region which, in its turn, will lead to the insufficiency of workers in the region under review and, consequently, will encourage the local companies to increase the wages. On the other hand, a positive spatial correlation may not exist or be insignificant, for example, in case of the obstacles for interregional migration of labor force.

The article has conducted a spatial-autoregressive analysis of interconnectedness of regional wages in the industrial context that answers the question if the wages will go up in the region in the specific industry in the context of increased wages in the neighboring region on condition of other similar conditions. The modelling has allowed evaluating the influence of some economic and social factors on the wages. These factors include: education level, percentage of pensioners, coefficient of demographic load, level of infrastructural development in the region, level of unemployment, labor performance, etc. The main novel-

\footnotetext{
1 Artur R. Nagapetyan, Senior Lecturer, department of economic Sciences, School of Economics and Management, Far Eastern Federal University, Vladivostok, Russia.

2 Danil V. Grishin, student, School of Economics and Management, Far Eastern Federal University, Vladivostok, Russia.

${ }^{3}$ Anna V. Kedrovskaya, student, School of Economics and Management, Far Eastern Federal University, Vladivostok, Russia.

For citing: Nagapetyan A. R., Grishin D. V., Kedrovskaya A. V. Spatial-autoregressive analysis of interregional interconnectedness of wages in the context of industry // PACIFIC RIM: Economics, Politics, Law. 2020. No. 1. P. 46-60.
} 
ty of the research lies in applying the models of spatial auto-regression that, in addition to revealing the spatial interconnections, give more precise evaluation of coefficients which describe the influence of these factors on the wages as they can partially solve the existing problems connected with the existence of omitted variables in the classic regressive models. Our analysis required reviewing the corresponding regional data from 2010 to 2016 for 83 subjects of the Russian Federation on the following industries: agriculture, hunting and forestry; food manufacturing (including beverages) and tobacco; construction; wholesale including trading via agents; government administration and military security; processing of timber and manufacturing of wood products (except for the furniture). A positive value of spatial correlation was discovered for all industries under research except for the wholesale trading and timber processing. The obtained results and conclusions can further be used by government administrative bodies in realization of measures of social and economic policies aimed at labor market regulation including the measures of attracting and retaining the labor force at the specific territory.

Key words: labor force migration, differentiation of income, model with fixed effects, interregional dependence of wages, models with spatial effects, real wages, spatial interrelation, spatial sampling, spatial-autoregressive analysis, spatial effects, regional wages in the industry, regional factors, social policy, economic security of the country.

Важной причиной оттока трудоспособного населения, помимо низкого уровня развитости инфраструктуры, является более низкий уровень заработных плат по сравнению с другими регионами. Для регионов Дальневосточного федерального округа эта тема представляется актуальной, поскольку каждый год здесь наблюдается отрицательный миграционный прирост. Только в 2018 г. отток составил 36,7 тыс. человек [2]. Основное направление переезда - это Центральный федеральный округ, где наблюдается относительно более высокий уровень развития инфраструктуры и при этом более низкая стоимость потребительской корзины.

Существует ли вообще межрегиональная взаимосвязанность между величинами заработных плат? Может ли исследование данной взаимосвязи помочь объяснить, почему в одних отраслях миграция труда происходит сильнее, чем в других. Последнее может быть важным основанием для принятия управленческих решений в сфере регулирования рынка труда, особенно в межотраслевом разрезе.

Исследовательская проблема: произойдёт ли повышение заработной платы в регионе, если в соседних регионах заработная плата повысилась? Во-первых, возможно наличие положительной пространственной корреляции (повышение оплаты труда в соседнем регионе приведёт к тому, что рабочие станут уезжать в этот регион, что, в свою очередь, приведёт к дефициту рабочих в родном регионе, где компании в таком случае будут вынуждены повышать оплату труда). Во-вторых, связь 
может отсутствовать в случае наличия тех или иных барьеров, препятствующих переезду из одного региона в другой. Тем самым данное исследование направлено на то, чтобы выявить наличие или отсутствие пространственной связи между уровнем оплаты труда как в среднем по России, так и в отдельно взятой отрасли.

Развитие всё более сложных эконометрических подходов позволяет заново пересмотреть существующие подходы к моделированию уровня региональных заработных плат. Так, в недавнем исследовании в ходе оценивания модели Оукена была продемонстрирована значимость учёта пространственных взаимосвязей между регионами на примере Российских данных [3]. Последнее позволило избежать проблем, связанных со смещённостью и недооценкой коэффициента Оукена в стандартной модели. Помимо возможности выявления межрегиональных взаимосвязей, пространственно-авторегрессионный анализ позволяет частично решить известные проблемы, связанные с наличием пропущенных переменных в классических регрессионных моделях.

В ходе анализа литературы были выявлены следующие факторы, влияющие на уровень заработной платы в регионе. Согласно стандартной микроэкономической теории, существуют два основных фактора - это производительность труда и безработица [4]. Исходя из теоретической концепции занятости, между безработицей и оплатой труда существует отрицательная взаимосвязь. Среди экономистов существует такое объяснение механизма безработицы: при высоком уровне безработицы уменьшается переговорная сила работников, это означает то, что, если работники в процессе переговоров станут требовать повышения заработной платы, фирма не пойдет на уступки, а примет на работу более дешёвую рабочую силу, так как в данный момент на рынке труда переизбыток свободных трудовых ресурсов. Говоря о влиянии производительности труда, можно отметить, что наблюдается положительная взаимосвязь данного показателя и оплаты труда, что вполне объяснимо, поскольку работник, производящий в единицу времени больше продукции, чем остальные, получает и более высокую заработную плату.

Говоря о производительности труда, необходимо также упомянуть концепцию «Эффективных заработных плат». Исходя из данной концепции, компания, заинтересованная в высокой производительности труда, может стратегически устанавливать более высокие ставки заработной платы для того, чтобы работники стали работать эффективнее. То есть, в таком случае наблюдается обратная взаимосвязь: чем выше заработная плата, тем выше производительность. Вакуленко и Гурвич, проверяя гипотезу о зависимости производительности труда от величины заработной платы на российских данных посредством проведения теста причинности по Гренджеру, не обнаружили значимых взаимосвязей между рассматриваемыми факторами [1]. В том же исследовании были эмпирически подтверждены теоретические концепции о механизмах влияния производительности и безработицы на раз- 
мер заработной платы с помощью эмпирических данных рынка труда РФ посредством эконометрического моделирования. Проведя моделирование связей между основными переменными рынка труда, авторы получили ключевые показатели оценки коэффициентов, которые не противоречат экономической логике. То есть между оплатой труда и безработицей наблюдается отрицательная взаимосвязь, а между оплатой труда и производительностью - положительная.

Следующим фактором, который обычно используется при моделировании величин заработных плат, является образование. Согласно теоретическим представлениям, зарплата находится в положительной зависимости от уровня образования [5]. Механизм влияния этого фактора вполне логичен, ведь человек, получивший высшее образование, имеет более высокие компетенции по сравнению с человеком без образования. Следовательно, первый ценится больше, чем второй, и получает своеобразную «премию» за высшее образование.

B исследовании Gatti et al. было показано, что уровень развития финансового рынка способствует увеличению занятости [6]. В свою очередь, увеличивается заработная плата посредством снижения безработицы. В настоящей работе также будет оцениваться зависимость уровня заработных плат для разных отраслей в регионе от уровня развитости финансовой инфраструктуры.

Как же влияет на уровень региональных заработных плат такой фактор, как доля населения старше трудоспособного возраста? С одной стороны, производительность таких работников падает, поэтому должна падать и их заработная плата. С другой стороны, компании относятся к таким рабочим как к опытным специалистам, следовательно зарплата в таком случае будет либо оставаться на одном уровне, либо повышаться. В работе Ours et al. было обнаружено отсутствие взаимосвязи между оплатой труда и долей возрастных работников [7]. В это же время в исследовании Hellerstein et al. демонстрируется наличие прямой взаимосвязи [8]. В настоящей работе рассматриваемый фактор используется при моделировании уровня региональных отраслевых заработных плат.

По итогам проведённого анализа литературы были определены основные факторы влияния на заработную плату. К их числу относятся уровень образованности, доля пенсионеров, коэффициент демографической нагрузки, уровень развития финансовой инфраструктуры в регионе, уровень безработицы, производительность труда.

Основной новизной работы представляется использование моделей пространственной авторегрессии, что помимо выявления пространственных взаимосвязей позволило получить более точные оценки коэффициентов, характеризующих влияние на заработную плату рассмотренных факторов ввиду того, что частично удаётся решить известные проблемы, связанные с наличием пропущенных переменных в классических регрессионных моделях [9]. 


\section{Исследовательские гипотезы}

Исходя из предположения о том, что российский рынок труда имеет неоднородную структуру, были выдвинуты гипотезы:

1. Показатели пространственной связи в отраслевом разрезе имеют значительные различия;

2. Оценки коэффициентов рассмотренных факторов, влияющих на уровень оплаты труда, имеют значительные различия в отраслевом разрезе.

\section{Данные и модели}

В работе используются данные о социально-экономических факторах, взятых из официальных статистических источников [10]. В таблице 1 приведена информация о переменных, которые были использованы при моделировании региональных отраслевых заработных плат.

Таблица 1

Информация о переменных, которые были использованы при моделировании региональных отраслевых заработных плат

\begin{tabular}{|l|l|l|}
\hline Обозначение & \multicolumn{1}{|c|}{ Фактор } & \multicolumn{1}{c|}{ Способ расчета } \\
\hline $\mathrm{Y}$ & $\begin{array}{l}\text { Реальная заработная } \\
\text { плата }\end{array}$ & Через индекс потребительских цен (100=2012) \\
\hline $\mathrm{X} 1$ & Уровень образованности & Доля населения с высшим образованием \\
\hline $\mathrm{X} 2$ & Доля пенсионеров & $\begin{array}{l}\text { Доля населения старше трудоспособного } \\
\text { возраста }\end{array}$ \\
\hline $\mathrm{X} 3$ & $\begin{array}{l}\text { Коэффициент } \\
\text { демографической } \\
\text { нагрузки }\end{array}$ & $\begin{array}{l}\text { Сколько лиц нетрудоспособных возрастов } \\
\text { приходится на 1000 человек трудоспособного } \\
\text { возраста }\end{array}$ \\
\hline Х5 & $\begin{array}{l}\text { Уровень развития фи- } \\
\text { нансовой инфраструкту- } \\
\text { ры в регионе }\end{array}$ & $\begin{array}{l}\text { Отношение числа кредитных организаций } \\
\text { и их филиалов в субъекте к числу } \\
\text { трудоспособных }\end{array}$ \\
\hline Х6 & $\begin{array}{l}\text { Уровень безработицы } \\
\text { По данным выборочных обследований } \\
\text { рабочей силы в среднем за год }\end{array}$ \\
\hline & $\begin{array}{l}\text { Производительность } \\
\text { труда }\end{array}$ & $\begin{array}{l}\text { Отношение ВРП региона к среднегодовому } \\
\text { количеству занятых }\end{array}$ \\
\hline
\end{tabular}

Для проверки сформированных гипотез будет использоваться обычная модель с фиксированными эффектами (1) и модель с пространственно-авторегрессионными эффектами и фиксированными эффектами (2).

$$
\begin{gathered}
Y_{\text {it }}=\alpha_{\mathrm{i}}+\beta_{1} * X 1_{\text {it }}+\beta_{2} * X 2_{\text {it }}+\beta_{3} * X 3_{\text {it }}+\beta_{4} * X 4_{\text {it }}+ \\
+\beta_{5} * X 5_{\text {it }}+\beta_{6} * X 6_{\text {it }}+\varepsilon_{\text {it }},
\end{gathered}
$$


Нагапетян А. Р., Гришин Д. В., Кедровская А. В. Пространственно-авторегрессионный анализ межрегиональной взаимосвязанности заработных плат в отраслевом разрезе

$$
\begin{gathered}
Y_{\mathrm{it}}=\alpha_{\mathrm{i}}+\rho * \mathrm{~W} * Y_{\mathrm{it}}+\beta_{1} * X 1_{\mathrm{it}}+\beta_{2} * X 2_{\mathrm{it}}+\beta_{3} * X 3_{\mathrm{it}}+ \\
+\beta_{4} * X 4_{\mathrm{it}}+\beta_{5} * X 5_{\mathrm{it}}+\beta_{6} * X 6_{\mathrm{it}}+\varepsilon_{\mathrm{it}},
\end{gathered}
$$

где величина $\alpha_{\mathrm{i}}$ выражает индивидуальный эффект объекта i, не зависящий от времени $\mathrm{t}$, при этом регрессоры не содержат константу; $\mathrm{W}$ - матрица, характеризующая пространственную компоненту в модели; $\rho$ - коэффициент, отражающий наличие пространственных эффектов; $\mathrm{Y}_{\mathrm{it}}$ - реальная заработная плата по отраслям.

\section{Результаты}

Расчёты проводились по данным за период с 2012 г. по 2016 г. с использованием статистического пакета STATA. Изначально было отобрано 83 региона, в расчётах использовалась информация по 63. Выборка была упрощена, чтобы не допустить смещения из-за наличия аутлайеров. В таблице 2 представлены описательные статистики по основным переменным до обработки данных и после обработки.

Таблица 2

\section{Описательные статистики основных переменных}

\begin{tabular}{|c|c|c|c|c|c|c|}
\hline \multicolumn{7}{|c|}{ До обработки } \\
\hline Переменные & Obs & Mean & Std. Dev. & Min & Max & Var, \% \\
\hline $\mathrm{Y}$ & 83 & 23797.15 & 10881.81 & 14984.29 & 67312.3 & 45.73 \\
\hline $\mathrm{X} 1$ & 83 & 31.07 & 4.79 & 21.9 & 48.5 & 15.42 \\
\hline $\mathrm{X} 2$ & 83 & 24.04 & 4.73 & 10 & 30.2 & 19.68 \\
\hline $\mathrm{X} 3$ & 83 & 768.65 & 64.05 & 536 & 914 & 8.33 \\
\hline $\mathrm{X} 4$ & 83 & 0.026 & 0.013 & 0.01 & 0.06 & 50.00 \\
\hline $\mathrm{X} 5$ & 83 & 6.78 & 3.77 & 1.6 & 30.2 & 55.60 \\
\hline $\mathrm{X} 6$ & 83 & 699.88 & 721.99 & 235.66 & 5624.41 & 103.16 \\
\hline \multicolumn{7}{|c|}{ После обработки } \\
\hline Переменные & Obs & Mean & Std. Dev. & Min & Max & Var \\
\hline $\mathrm{Y}$ & 63 & 20484.33 & 3997.55 & 14984.29 & 33829.78 & 19.52 \\
\hline $\mathrm{X} 1$ & 63 & 30.48 & 4.03 & 21.9 & 42.4 & 13.22 \\
\hline $\mathrm{X} 2$ & 63 & 26.03 & 2.43 & 19.7 & 30.2 & 9.34 \\
\hline $\mathrm{X} 3$ & 63 & 789.38 & 41.15 & 699 & 914 & 5.21 \\
\hline $\mathrm{X} 4$ & 63 & 0.025 & 0.011 & 0.01 & 0.05 & 44.00 \\
\hline $\mathrm{X} 5$ & 63 & 5.9 & 1.65 & 1.6 & 9.9 & 27.97 \\
\hline $\mathrm{X} 6$ & 63 & 535.08 & 141.77 & 293.17 & 919.36 & 26.50 \\
\hline
\end{tabular}
до обработки данных и после обработки

Источник: выполнено авторами статьи с использованием программы Stata 
В данной таблице можно также наблюдать коэффициент вариации в крайнем правом столбце, значение которого уменьшилось после обработки данных.

Далее переходим непосредственно к эконометрическому анализу влияния рассматриваемых факторов на отраслевые заработные платы. В таблице 3 приведены наименования и обозначения рассматриваемых в работе отраслей.

Таблиияа 3

Наименования и обозначения рассматриваемых в работе отраслей

\begin{tabular}{|l|l|}
\hline Y & Все отрасли \\
\hline$Y_{\text {AGR }}$ & Сельское хозяйство, охота и лесное хозяйство \\
\hline$Y_{\text {FOOD }}$ & Производство пищевых продуктов (включая напитки) и табака \\
\hline$Y_{\text {BUILD }}$ & Строительство \\
\hline$Y_{\text {WHOLESE }}$ & Оптовая торговля, включая торговлю через агентов \\
\hline$Y_{\text {RETAIL }}$ & Розничная торговля; ремонт бытовых и личных изделий \\
\hline$Y_{\text {STATE }}$ & Гос. управление и военная безопасность; соц. обеспечение \\
\hline$Y_{\text {WOOD }}$ & Обработка древесины и производство изделий из дерева (кроме мебели) \\
\hline
\end{tabular}

Источник: выполнено авторами статьи

Для наглядного восприятия данных была построена карта, отображающая информацию о заработной плате в регионах. Была взята заработная плата за 2016 г. (рис. 1). Визуальный анализ показывает наличие пространственной корреляции, поскольку регионы с высокой заработной платой тянутся к регионам с высокой заработной платой и, соответственно, наоборот.

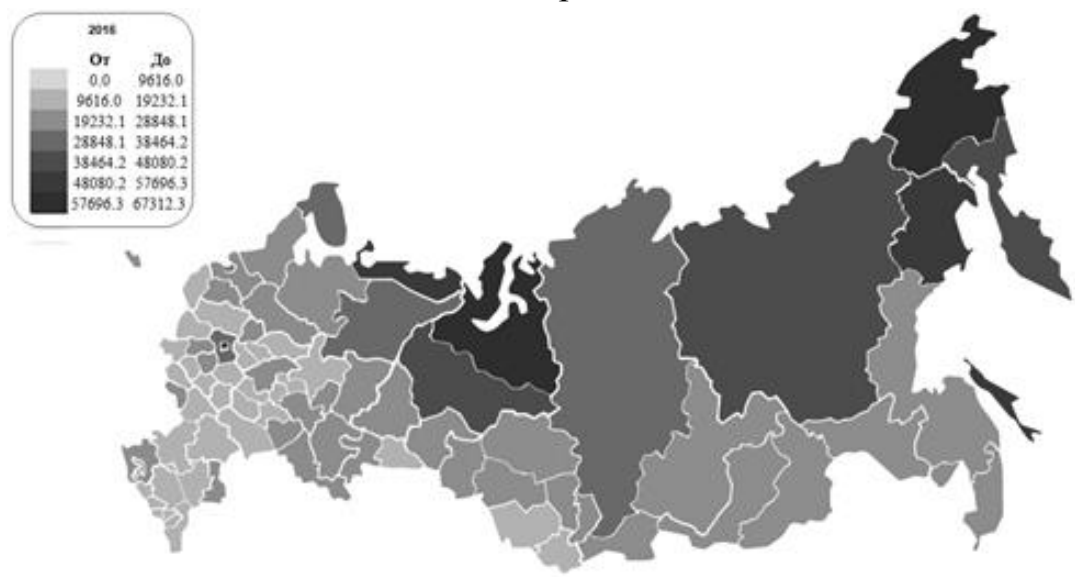

Puc. 1. Реальная заработная плата в регионах России в 2016 г.

Источник: выполнено авторами статьи 
Нагапетян А. Р., Гришин Д. В., Кедровская А. В. Пространственно-авторегрессионный анализ межрегиональной взаимосвязанности заработных плат в отраслевом разрезе

Таблийа 4

Результаты оценивания модели (1) для рассматриваемых отраслей

\begin{tabular}{|c|c|c|c|c|c|c|c|c|}
\hline VARIABLES & $\mathrm{Y}$ & $\mathrm{Y}_{\mathrm{AGR}}$ & $\mathrm{Y}_{\mathrm{FOOD}}$ & $\mathrm{Y}_{\text {BUILD }}$ & $\mathrm{Y}_{\text {WHOLESALE }}$ & $Y_{\text {RETAIL }}$ & $Y_{\text {STATE }}$ & $Y_{\text {WOOD }}$ \\
\hline $\mathrm{X} 1$ & $38^{*}$ & -9 & -11 & -44 & -44 & 1 & 48 & 34 \\
\hline $\mathrm{X} 2$ & $1394 * * *$ & 2289 **** & $1676^{* * * *}$ & 466 & 573 & 1489 **** & $2494 * * *$ & $2603 * *$ \\
\hline $\mathrm{X} 3$ & $-47 * * *$ & $-40^{* * * *}$ & $-35^{* * * *}$ & $-45^{* * * *}$ & -19 & $-33^{* * * *}$ & $-103 * * *$ & 4 \\
\hline $\mathrm{X} 4$ & $-32267 * * *$ & $-29279 * * * *$ & 2720 & $-37077^{*}$ & -1561 & -11131 & -17879 & -8013 \\
\hline $\mathrm{X} 5$ & $-289^{* * *}$ & -151 & $-278 * *$ & -269 & -73 & -120 & $-808 * * *$ & 331 \\
\hline $\mathrm{X} 6$ & $6 * * *$ & $6 * * *$ & 1 & $7 * *$ & 3 & $4 * *$ & $9 * *$ & 1 \\
\hline Constant & 19394*** & $-14201 * *$ & 3042 & $42205^{* * * *}$ & 16723 & 125 & $43125^{* * * * *}$ & $-53827 * * * *$ \\
\hline Observations & 315 & 315 & 315 & 315 & 315 & 315 & 315 & 315 \\
\hline R-squared & 0.467 & 0.272 & 0.106 & 0.435 & 0.078 & 0.094 & 0.678 & 0.56 \\
\hline $\begin{array}{l}\text { Number } \\
\text { of Region }\end{array}$ & 63 & 63 & 63 & 63 & 63 & 63 & 63 & 63 \\
\hline AIC & 4987 & 5070 & 5180 & 5467 & 5432 & 5099 & 5489 & 5936 \\
\hline BIC & 5013 & 5096 & 5206 & 5494 & 5458 & 5126 & 5516 & 5963 \\
\hline
\end{tabular}

Источник: выполнено авторами статьи с использованием программы Stata

В таблице 4 приведены результаты оценивания модели (1) для рассматриваемых отраслей. Данная таблица формируется автоматически в программе Stata. В верхней строке перечислены зависимые переменные. Далее следуют оценки коэффициентов соответствующих моделей. Большинство коэффициентов значимы и влияют согласно теоретической концепции. Исключением является переменная X1 (доля людей с высшим образованием), которая влияет только в том случае, если смотреть в среднем по России, в других отраслях влияния не оказывает. Также уровень развития финансовой инфраструктуры отрицательно влияет на заработную плату.

В оставшихся строках таблицы содержится информация об основных характеристиках моделей. Это, соответственно, количество наблюдений, R-квадрат, число регионов, задействованных в расчетах, и информационные критерии AIC и BIC.

В таблице 5 приведены результаты оценивания модели (2) для рассматриваемых отраслей. Исходя из анализа результатов оценивания модели в отраслевом разрезе, следует, что такие факторы, как уровень образованности и уровень безработицы, в большинстве из рассмотренных отраслей не оказывают значимое влияние на реальную заработную плату. Поэтому, соответственно, и в общем случае, когда в качестве зависимой переменной рассматривалась средняя заработная плата по региону, эти два фактора не будут значимыми. Показатель доли пенсионеров в пяти отраслях из семи исследованных влияет значимо положительно на заработную плату. Поэтому в общем случае влияние данного фактора соответствует общей тенденции. Коэффициент демографической нагрузки в 5 отраслях из 7 влияет значимо отрицательно, и в общей модели повторяется данная тенденция. Уровень развития финансовой инфраструктуры значим отрицательно в отрасли сельского хозяйства и значим положительно в отрасли государственного управления. В остальных отрас- 
лях показатель не оказывает влияния на заработную плату. Показатель производительности труда влияет положительно всего в трёх отраслях. Несмотря на то, что это составляет менее $50 \%$ от исследованных отраслей, в общей модели производительность труда также влияет значимо положительно на заработную плату.

Таблица 5

Результаты оценивания модели (2) для рассматриваемых отраслей

\begin{tabular}{|c|c|c|c|c|c|c|c|c|}
\hline $\begin{array}{c}\text { VARIABL } \\
\text { ES }\end{array}$ & $\mathrm{Y}$ & $\mathrm{Y}_{\mathrm{AGR}}$ & $\mathrm{Y}_{\mathrm{FOOD}}$ & $\mathrm{Y}_{\text {BUILD }}$ & $\begin{array}{c}\text { Y }_{\text {WHOLESA }} \\
\text { LE }\end{array}$ & $Y_{\text {RETAIL }}$ & $\mathrm{Y}_{\text {STATE }}$ & $Y_{\text {WOOD }}$ \\
\hline $\mathrm{X} 1$ & 6 & -10 & -26 & -54 & -43 & -2 & $-46^{* * *}$ & 34 \\
\hline $\mathrm{X} 2$ & $1310^{* * *}$ & $2346^{* * *}$ & $1720 * * *$ & 536 & 615 & $1492 * * *$ & $2424 * * *$ & $2584 * * *$ \\
\hline X3 & $-29 * * *$ & $-43 * * *$ & $-31 * * *$ & $-27 *$ & -17 & $-31 * * *$ & $-42 * * *$ & -1 \\
\hline $\mathrm{X} 4$ & $-10601 * *$ & $-26837 * * *$ & 6932 & -27165 & 871 & -5641 & $21623 * *$ & -6311 \\
\hline $\mathrm{X} 5$ & -61 & -136 & $-177 *$ & -155 & -65 & -78 & $-219 * *$ & 329 \\
\hline X6 & $3 * * *$ & $6 * * *$ & -1 & $5 *$ & 2 & $3 * *$ & 1 & 1 \\
\hline rho & $0.9 * * *$ & $0.3 * *$ & $0.6 * * *$ & $0.6^{* * *}$ & 0.2 & $0.4 * * *$ & $0.9 * * *$ & 0.1 \\
\hline Observations & 315 & 315 & 315 & 315 & 315 & 315 & 315 & 315 \\
\hline R-squared & 0.015 & 0.073 & 0.039 & 0.142 & 0.037 & 0.074 & 0.064 & 0.002 \\
\hline $\begin{array}{l}\text { Number } \\
\text { of Region }\end{array}$ & 63 & 63 & 63 & 63 & 63 & 63 & 63 & 63 \\
\hline AIC & 4688 & 5069 & 5156 & 5449 & 5432 & 5090 & 5062 & 5938 \\
\hline $\mathrm{BIC}$ & 4718 & 5099 & 5186 & 5479 & 5462 & 5120 & 5092 & 5969 \\
\hline
\end{tabular}

Источник: выполнено авторами статьи с использованием программы Stata

\section{Обсуждение результатов}

Согласно описанным выше результатам можно сделать вывод о том, что гипотеза о наличии значительных различий в коэффициенте пространственной взаимосвязанности была подтверждена. Отрасль с самым высоким показателем rho это отрасль государственного управления, военной безопасности и социального обеспечения. В данном случае rho равен 0,939. В целом, это может свидетельствовать о том, что, возможно, сотрудники данной отрасли не имеют особых барьеров для перемещения между регионами в поисках хорошей зарплаты. Другая интерпретация заключается в том, что большинство параметров в сфере оплаты труда в перечисленных сферах деятельности формируется централизованно, что и определяет их взаимосвязанность. В этой связи можно говорить о качестве сформированной модели, позволяющей обнаружить данную закономерность в данных. В свою очередь, это подразумевает то, что инфраструктура данной отрасли развита повсеместно в стране и не требует каких-либо особых мер поддержки для развития - в контексте удержания кадров. Вопросы, связанные с их квалификацией, выходят за рамки настоящего исследования.

Самое низкое значение rho, равное 0,306, имеет отрасль сельского хозяйства. Если рассматривать основную рабочую силу сельского хозяйства, то это рабочие, 
проживающие в сельской местности и привязанные к земле. Таким образом, можно судить о низком уровне развития инфраструктуры данной отрасли, а также о наличии затруднений с перемещением рабочих из региона в регион. Интерпретация заключается в том, что уровень развития рыночных отношений, в том числе на рынке труда данной отрасли, относительно неразвит и поэтому рост заработной платы в соседних регионах у селян имеет относительно более низкий уровень влияния на соответствующие показатели в самом регионе. Важную роль здесь играет и сам факт «привязанности к земле». Но это лишь говорит о том, что в этой связи у соответствующих категорий населения возникают сильные стимулы к переезду, в том числе в другой регион. Это, в свою очередь, создаёт предпосылки для приоритизации в контексте принимаемых мер поддержки. При этом речь может идти как о субсидировании сельскохозяйственной отрасли, так и просто о переобучении кадров и переориентации их на деятельность в другие сферы экономики.

Также можно рассмотреть отрасль оптовой торговли. Показатель rho в данном случае незначим, это свидетельствует о том, что в плане перетоков трудовых ресурсов имеются явные особенности, которые необходимо учитывать при регулировании рынка труда. В частности, речь идёт о повышенных стимулах к переезду у этой категории населения, ведь низкий показатель rho говорит о том, что рост заработной платы в данной отрасли в соседних регионах не приводит к росту в рассматриваемом регионе. Можно предложить разные интерпретации, почему так происходит, однако сам по себе этот результат демонстрирует наличие рисков по закреплению данной категории населения на территории.

Выдвинутая ранее гипотеза 2 также оказалась подтверждённой. Следовательно, это может указывать на значительный уровень неоднородности показателей межрегиональной взаимосвязанности заработных плат в отраслевом разрезе. Например, производительность труда в сельском хозяйстве имеет коэффициент $5.90^{* * *}$, в то же время в отрасли государственного управления данный показатель незначим. Это вполне объяснимо, поскольку при повышении производительности рабочий за то же время выполняет больше работы и, соответственно, получает больше зарплату. В сельском хозяйстве, судя по всему, отдача от повышения работником эффективности своего труда больше, возможно, ввиду того, что данная отдача в большей степени зависит от показателей капиталовооружённости труда.

Интерес вызывает уровень безработицы, поскольку, согласно теории, данный фактор должен отрицательно значимо влиять на уровень оплаты труда. В рамках данной работы данный фактор не значим - как в большинстве отраслей, так и в среднем по России. Одной из возможных причин данного явления может быть низкое значения показателя текучести кадров, что, в свою очередь, и определяется уровнем развития рыночных отношения между работодателями и рабочими. То есть отрасль не может реагировать на изменения предложения на рынке труда 
уменьшением заработных плат ввиду наличия долгосрочных жёстких контрактов. В этой связи более правдоподобной видится интерпретация, связанная с существованием квалификационных требований. Речь идёт о способности рынка труда удовлетворять спрос на труд за счёт существующих безработных, что во многом зависит от существующих возможностей переквалификации и переобучения. Возможны и другие причины.

\section{Заключение}

Результатом выполнения работы стало подтверждение обеих гипотез, поставленных ранее. Во-первых, о том, что показатель пространственной корреляции имеет значительные различия в разных отраслях; во-вторых, - оценки влияния факторов на заработную плату также являются различными в разных отраслях.

Можно сказать, что удалось в некоторой степени оценить неоднородность региональных отраслевых рынков труда. Это важно, поскольку результаты данного исследования могут быть полезными при приоритизации категорий населения с точки зрения мер по их закреплению на территории. Ключевое предложение заключается в том, чтобы учитывать величину коэффициентов межрегиональной взаимосвязанности заработных плат в отраслевом разрезе. При этом чем выше коэффициент, тем ниже риски миграции данной категории населения при прочих равных условиях. Несомненным преимуществом является объективность данного метода, что позволит принимать более эффективные управленческие решения. Полученные данные о влиянии социально-экономических факторов могут выступать в роли рычагов, позволяющих корректировать уровень заработной платы в конкретных отраслях в регионе либо принимать решение о переквалификации и переобучении персонала и т.д. В дальнейших исследованиях предстоит напрямую проверить гипотезу о влиянии коэффициентов межрегиональной взаимосвязанности заработных плат в отраслевом разрезе на склонность к миграции.

\section{Список литературы}

1. Вакуленко, Е. С. Моделирование механизмов российского рынка труда / Е. С. Вакуленко, Е. Т. Гурвич // Вопросы экономики. - 2015. - № 11. - С. 5-29.

2. Внутрироссийская миграция по территориям прибытия и выбытия («шахматка» по федеральным округам). 2018. - URL: http://old.gks.ru/wps/wcm/connect/ rosstat_main/rosstat/ru/statistics/population/demography/\# (дата обращения: 13.05.2020).

3. Вакуленко, Е. С. Анализ связи между региональными рынками труда в России с использованием модели Оукена // Прикладная эконометрика. - 2015. № 4. - С. 28-48.

4. Blanchard, O. Wage dynamics: reconciling theory and evidence / O. Blanchard, L. Katz // American Economic Review. - 1999. - No. 89. - P. 69-74. 
5. Ощепков, А. Ю. Отдача от высшего образования в российских регионах // Экономический журнал Высшей школы экономики. - 2010. - Т. 14, вып. 4. C. $468-491$.

6. Gatti, D. Unemployment and finance: how do financial and labour market factors interact? / D. Gatti, C. Rault, A.-G. Vaubourg // Oxford Economic Papers. - 2011. Vol. 64, iss. 3. - P. 464-489.

7. Ours, J. C. Age, Wage and productivity in Dutch manufacturing / J. C. Ours, L. Stoeldraijer // Economist. - 2011. - Vol. 159, iss. 2. - P. 113-137.

8. Hellerstein, J. K. Wages, productivity, and worker characteristics: Evidence from plant-level production functions and wage equations / J. K. Hellerstein, D. Neumark, K. R. Troske // Journal of Labor Economics. - 1999. - Vol. 17, iss. 3. - P. 409-446.

9. Демидова, О. А. Пространственно-авторегрессионная модель для двух групп взаимосвязанных регионов (на примере восточной и западной части России) // Прикладная эконометрика. - 2014. - Т. 34, № 2. - С. 19-35.

10. Регионы России. Социально-экономические показатели. 2017. - URL: https://www.gks.ru/folder/210/document/13204 (дата обращения: 13.05.2020).

\section{References}

1. Vakulenko E. S., Gurvich E. T. Modelirovanie mekhanizmov rossiiskogo rynka truda [Modeling of the mechanisms of the Russian labor market]. Voprosy ekonomiki, 2015, no. 11, pp. 5-29.

2. Internal Russian migration in the territories of arrival and departure («chess» in federal districts). 2018. Available at: http://old.gks.ru/wps/wcm/connect/rosstat_ma in/rosstat/ru/statistics/population/demography/\# (accessed 13 May 2020). (In Russian).

3. Vakulenko E. S. Analiz svyazi mezhdu regional'nymi rynkami truda v Rossii $\mathrm{s}$ ispol'zovaniem modeli Oukena [Analysis of the relationship between regional labor markets in Russia using the Ouken model]. Prikladnaya ekonometrika, 2015, no. 4, pp. $28-48$.

4. Blanchard O., Katz L. Wage dynamics: reconciling theory and evidence. American Economic Review, 1999, no. 89, pp. 69-74.

5. Oshchepkov A. Yu. Otdacha ot vysshego obrazovaniya v rossiiskikh regionakh [Return on higher education in the Russian regions]. Ekonomicheskii zhurnal Vysshei shkoly ekonomiki, 2010, vol.14, iss. 4, pp. 468-491.

6. Gatti D, Rault C., Vaubourg Anne-Gaël. Unemployment and finance: how do financial and labour market factors interact? Oxford Economic Papers, 2011, vol. 64, iss. 3, pp. 464-489.

7. Ours J.C., Stoeldraijer L. Age, wage and productivity in Dutch manufac-turing. Economist, 2011, vol. 159, iss. 2, pp. 113-137. 
8. Hellerstein J. K., Neumark D., Troske K. R. Wages, productivity, and worker characteristics: Evidence from plant-level production functions and wage equations. Journal of Labor Economics, 1999, vol. 17, iss. 3, pp. 409-446.

9. Demidova O. A. Prostranstvenno-avtoregressionnaya model' dlya dvukh grupp vzaimosvyazannykh regionov (na primere vostochnoi i zapadnoi chasti Rossii) [Spatialautoregressive model for two groups of interconnected regions (on the example of the eastern and western parts of Russia). Prikladnaya ekonometrika, 2014, vol. 34, no. 2, pp. 19-35.

10. Regions of Russia. Socio-economic indicators. 2017. Available at: https://www.gks.ru/folder/210/document/13204 (accessed 13 May 2020). (In Russian). 